\title{
The effect of intradermal administration of inactive platelet- rich plasma on flap viability in rats ${ }^{1}$
}

\author{
Erkan Orhan', Ahmet Çağrı Uysal", Engin Başer'"I, Dürdane Keskinv, Çiğdem Demiroğlu-Yakut
}

'Assistant Professor, Department of Plastic Surgery, Namik Kemal University, Tekirdağ, Turkey. Scientific, intellectual, conception and design of the study; acquisition, analysis and interpretation of data; technical procedures; statistical analysis; manuscript preparation and writing; final approval.

"Associate Professor, Department of Plastic Surgery, Başkent University, Ankara, Turkey. Scientific, intellectual, conception and design of the study; manuscript preparation and writing; final approval.

"'MD, Department of Otorhinolaryngology, Sultan I. Murat Public Hospital, Edirne, Turkey. Acquisition, analysis and interpretation of data; statistical analysis.

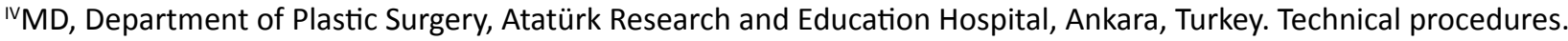
${ }^{\vee} M D$, Department of Plastic Surgery, Niğde Public Hospital, Niğde, Turkey. Technical procedures.

\begin{abstract}
Purpose: To evaluate the effect of inactive form of platelet rich plasma (PRP) on the flap viability.

Methods: Thirty six rats were used. Rats were divided into six groups then $9 \times 3 \mathrm{~cm}$ random pattern skin flaps were elevated from dorsum of all rats. For precluding vascularization from the base, a silicone layer was placed under the flap in groups 2(only flap+silicone), 4 (saline+silicone) and 6(PRP+silicone). In groups 1(only flap), 2(only flap+silicone) nothing was done except flap surgery. In groups 3 (saline) and 4 (saline+silicone), saline was applied intradermally, in groups 5(PRP) and 6(PRP+silicone), inactive form of PRP which obtained from different 16 rats was applied intradermally, into certain points of flaps immediately after surgery. After 7 days flap necrosis ratio was measured in all groups.

Results: Mean necrosis rate in group 5(PRP) (16.05\%) was statistically significantly lower than group 1 (only flap) $(31,93 \%)$ and group 3 (saline) $(30,43 \%)(p<0.001)$. Mean necrosis rate in group 6(PRP+silicone) (36.37\%) was statistically significantly lower than group 2(only flap+silicone) (47.93\%) and group 4(saline+silicone) $(45.65 \%)(p<0.001)$.

Conclusion: Intradermal inactive platelet rich plasma administration decreases flap necrosis so for skin application.
\end{abstract}

Key words: Platelet-Rich Plasma. Surgical Flaps. Skin. Rats. 


\section{Introduction}

Random pattern skin flaps are commonly used for the reconstruction of defects in plastic surgery but despite the development of flap surgery techniques in recent years, partial or complete flap necrosis is still an important complication, especially in the distal region of the flap ${ }^{1}$.

In the last 30 years, many studies have been performed to preclude necrosis and increase flap viability and many pharmacological agents such as, sympatholytics, vasodilators, prostaglandin inhibitors, anticoagulants, and free radical scavengers have been used experimentally in order to increase the viability of skin flaps ${ }^{2}$. However, a clinically useful treatment method hasn't been developed yet in this regard ${ }^{3}$.

Platelet-Rich Plasma (PRP) is a concentration in a small volume of plasma in platelets ${ }^{4}$. There are granules in platelets called alfa, beta and lambda granules and alfa granules contains many growth factors including epidermal growth factor (EGF), fibroblast growth factor (FGF), insulin-like growth factor (IGF), keratinocyte growth factor (KGF), plateletderived growth factor (PDGF), transforming growth factor-beta (TGF-b) and vascular endothelial growth factor (VEGF) $)^{5}$. PRP, with its high-level growth factor contents, is used clinically for bone and wound healing, oralmaxillofacial surgery, dental surgery and plastic surgery ${ }^{6,7}$.

Previously it was shown that PRP increase the viability of skin flaps in several experimental study ${ }^{1,8,9}$ and this effect of PRP is provided by growth factors in alfa granules. Degranulation of alfa granules is necessary for the secretion of growth factors from PRP and this is referred to as activation. In experimental study and also clinical application, PRP is activated by mixing with calcium(Ca) or thrombin ${ }^{5}$. However, by mixing with calcium(Ca) or thrombin, PRP becomes jelly and therefore it is not suitable for injection by needle. Because of this, special appartus should be used for application of PRP. Also, jelly form of PRP is not suitable for administration into skin as well into skin flap, so in previous experimental study and clinic use in cosmetic therapy, PRP was administered to subcutaneous level ${ }^{1,7}$.

We hypothesized that activation of PRP is not necessary for protective effect of PRP on skin, and inactive form of PRP which is applied into the dermis would have viability increasing effect on flap. The objective of this study was to evaluate whether inactive form of plateletrich plasma would increase skin flap survival in an experimental rat model.

\section{Methods}

This study was performed in Gazi University, Faculty of Medicine, Laboratory Animals and Experimental Research Center after receiving the approval of Gazi University, Experimental Animals Ethics Committee.

Fifty two Wistar-type female rats with weights between 200 and $300 \mathrm{~g}$, have been used in the study. PRP was obtained from 16 rats. The remaining 36 rats were kept in proper cages under conditions of $22 \pm 20^{\circ} \mathrm{C}$ and 12 hours dark and 12 hours light. Nutrition requirements of the animals were addressed regularly by giving standard laboratory food and water.

All surgical procedures were performed with intraperitoneal administration of $50 \mathrm{mg} /$ $\mathrm{kg}$ ketamine- $\mathrm{HCl}$ (Alfamine) and $10 \mathrm{mg} /$ $\mathrm{kg}$ xylazine $\mathrm{HCl}$ (Rompun ${ }^{\circ}$ ) for anesthesia by the same surgeon under sterilized conditions. After the surgical procedure, rats were kept in individual cages to preclude them from hurting each other.

\section{Preparation of Platelet-Rich Plasma (PRP)}

The required blood for separation from other blood components of the platelet to form PRP 
was obtained by whole body blood collection from 16 donor rats with Ketamine $\mathrm{HCl}$ (Alfamine $-\mathrm{IM}$ ) and Xylazine $\mathrm{HCl}$ (Rompun $-\mathrm{IM}$ ) anesthesia through cardiac puncture.

270.000 platelet/liter were detected when platelet count was performed in $120 \mathrm{ml}$ of blood obtained from the rats. Blood was placed into $3.8 \%$ sodium citrate containing tubes and centrifugation was performed. Tubes were centrifuged for 10 minutes at $2400 \mathrm{rpm}$ as described by Tobita et al. ${ }^{10}$. A thin layer (buffer coat) was placed between light yellow plasma supernatant and the red bottom layer. This middle layer and the upper plasma layer were transferred into different tubes. These tubes were centrifuged for a second time for 15 minutes at $3600 \mathrm{rpm}$. At the end of this procedure, platelet-poor plasma accumulated at the top. A total of $12 \mathrm{ml}$ of platelet concentrate was taken into 1-cc insulin syringes to be used for the experiment. The platelet count was measured to be 931.000 platelets/liters in the platelet concentrate prepared after centrifugation. Platelet count increased 3.5-fold.

\section{Surgical procedure}

All rats were anesthetized with intraperitoneal administration of $50 \mathrm{mg} / \mathrm{kg}$ ketamine- $\mathrm{HCl}$ (Alfamine ${ }^{\circ}$ ) and $10 \mathrm{mg} / \mathrm{kg}$ xylazine $\mathrm{HCl}$ (Rompun ${ }^{\circ}$ ). Their backs were shaved, and the rats were fixed in a prone position. Surgical area was cleaned with $10 \%$ povidone iodine solution and covered under sterilized conditions. From their back, random patern dorsal flap included panniculus carnosus of $9 \times 3 \mathrm{~cm}$ in size was elevated ${ }^{11} .3 \times 4.5 \mathrm{~cm}$ silicone layer was placed under the distal region of the flap in 18 animals that were determined randomly for precluding vascularization from the wound bed. The other 18 animals did not have any material under the flaps. Elevated flaps were sutured back with $4 / 0$ Vicryl. Surgical suture lines were cleaned with $10 \%$ povidone iodine solution. 18 rats with silicone layers under the flap were divided into three groups with each group having 6 rats and named as groups 2, 4 and 6. The other 18 rats without silicone were again randomly divided into three groups with each group having 6 rats and the groups were named as 1, 3 and 5 .

In group 1(only flap group) and group 2(only flap + silicone group) nothing was done except flap surgery. In group 3 (saline group) and group 4 (saline + silicone group) immediately after surgery, a total of 0.9 cc saline was injected intradermally into specified points. In group 5 (PRP group) and group 6 (PRP + silicone group) immediately after surgery, a total of 0.9 cc preprepared PRP was injected intradermally into specified points. PRP was injected inactivated form so PRP was not mixing with calcium(Ca) or thrombin for activating.

PRP and saline were injected intradermally to nine points that were marked between $5 \mathrm{~cm}$ distal of pedicle to $7 \mathrm{~cm}$ distal of pedicle and $0.5 \mathrm{~cm}$ medial of sides, to be $0.1 \mathrm{cc}$ to each point.

After seven days of flap surgey, all rats were euthanized with high dose of anesthesia (200 $\mathrm{mg} / \mathrm{kg}$ of pentobarbital). Photos of the flaps and necrosis areas on the backs of the rats were taken with Kodak Easy Share M320 digital camera from standard distance, and then computerized with Windows Picture Manger (Microsoft). Zones covered with scabs and dark zones were defined to be necrotic. By using digital analysis software (Photoshop CS; Adobe Systems, Inc., San Jose, Calif.) pixels of necrotic and and viable areas were counted so necrotic and total flap area could be measured then the necrotic area percentages (necrotic area / all flap area $x$ 100) were calculated.

\section{Statistical analysis}

Data analysis was performed in 'SPSS for Windows 11.5' package software. The normality of the distribution of continuous variables was evaluated with Shapiro-Wilk test 
and homogeneity of the variances with Levene test. Descriptive statistics were shown as mean \pm standard deviation. Two-Way Analysis of Variance (Two-Way ANOVA) was used for statistical evaluation. In case main effects of intradermal injections types were found to be significant, 'post hoc Tukey' test was used to determine the cause of intergroup differences.

Table 1 - Mean necrosis ratio of groups.

\begin{tabular}{lll} 
& & $\begin{array}{l}\text { Necrosis } \\
\text { Ratio(\%) } \\
\text { Mean } \pm \text { SD }\end{array}$ \\
\hline $\begin{array}{l}\text { Group 1 } \\
\text { (only flap group) }\end{array}$ & 6 & $31.93 \pm 3.28$ \\
$\begin{array}{l}\text { Group 2 } \\
\text { (only flap + silicone }\end{array}$ & 6 & $47.93 \pm 5.01$ \\
group) \\
$\begin{array}{l}\text { Group 3 } \\
\text { (saline group) }\end{array}$
\end{tabular}

${ }^{a} \mathrm{P}<0.001$ vs. group $1,{ }^{b} \mathrm{P}<0.001$ vs. group $2,{ }^{C} \mathrm{P}<0.001$ vs. group $3,{ }_{P}<0.001$ vs. group $4,{ }_{P}<0.001$ vs. group $3,{ }_{P}<0.001$ vs. group 4.

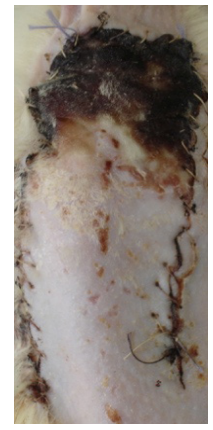

Group 1

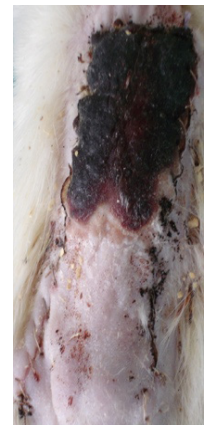

Group 2

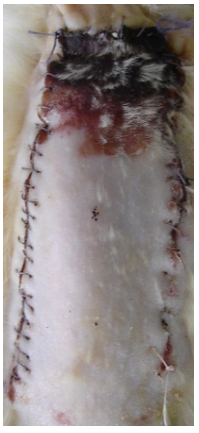

Group 3

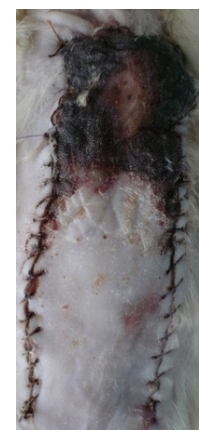

Group 4

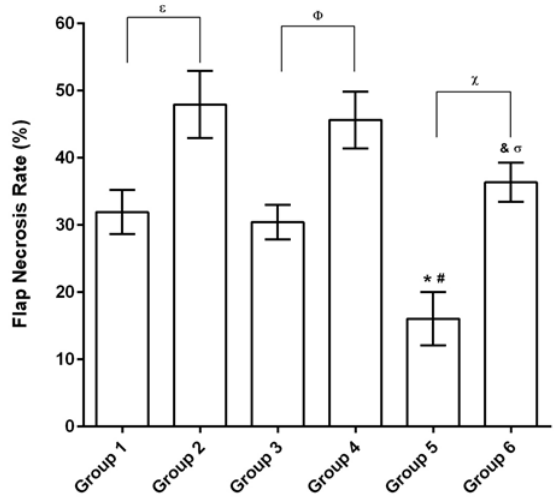

Figure 1 - Skin flap necrosis of the groups.

*Necrosis ratio in group 5 was statistically significantly lower than the group $1(p<0,001)$

\#Necrosis ratio in group 5 was statistically significantly lower than the group $3(p<0,001)$

\&Necrosis ratio in group 6 was statistically significantly lower than the group $2(p<0,001)$

opNecrosis ratio in group 6 was statistically significantly lower than the group $4(p<0,001)$

$\epsilon \mathrm{pNecrosis}$ ratio in group 1 was statistically significantly lower than the group $2(p<0,001)$

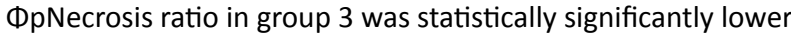
than the group $4(p<0,001)$

$\chi p$ Necrosis ratio in group 5 was statistically significantly lower than the group $6(p<0,001)$

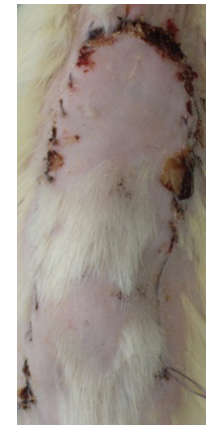

Group 5

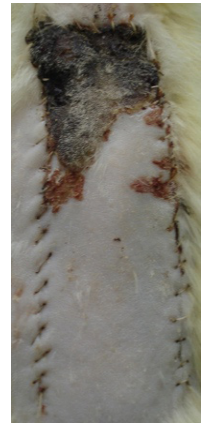

Group 6

Figure 2 - View of necrosis ratio of the flaps. 
Necrosis ratio in group 5 (PRP group) was statistically significantly lower than the group 1 (only flap group) and group 3(saline group) $(p<0.001$ and $p<0.001$, respectively), and there was no statistically significant difference in flap necrosis ratio between group 1(only flap group) and groups 3 (saline group). Necrosis ratio in group 6(PRP+silicone group) was statistically significantly lower than the group 2(only flap+silicone group) and group 4 (saline+silicone group) ( $p<0.001$ and $p<0.001$, respectively), and there was no statistically significant difference in flap necrosis ratio between group 2 (only flap+silicone group) and group 4(saline+silicone group). Necrosis ratio in group 5(PRP group) was statistically significantly lower than the group 6(PRP+silicone group) $(p<0.001)$, necrosis ratio in group 3(saline group) was statistically significantly lower than the group 4(saline+silicone group) $\quad(p<0.001)$ and necrosis ratio in group 1 (only flap group) was statistically significantly lower than the group 2(only flap+silicone group) $(p<0.001)$ (Table 1).

\section{- Discussion}

Previously a few studies have been carried out on the effect of PRP on flap circulation. In these studies, Findikcioglu et al. ${ }^{1}$ injected active form of PRP into subcutaneous layer of flap tissue one week before flap elevation, Li et al. ${ }^{8}$ and Wang et al. ${ }^{9}$ administered active form of PRP into subcutaneous layer of flap tissue after flap elevation and all of that studies showed that active form of PRP increased flap viability. Unlike these studies, we applied inactive form of PRP into dermal layer of flap tissue and showed that inactive form of PRP increased flap viability too.

In this study we used random patern dorsal skin flap model ${ }^{11}$ and we placed a silicone layer under the distal region of the flap in certain groups for inhibiting revascularization from the wound to demostrate flap viability increasing effect of inactive form of PRP is caused via revascularization from the wound bed or not. In random pattern skin flap models as we used, $52 \%$ part of the flap stays viable with basal vascularization as with composite graft $^{12}$ so revascularization from the wound bed is very important for flap viability. In all of the previous studies about active form of PRP and flap viability, PRPprovided flap viability increasing effect by promoting revascularization from the wound bed. In our study the least necrosis ratio was detected in the PRP group (group 5) but necrosis ratio in the PRP + silicone group (group 6) was statistically significantly upper than only flap group (group 1) and salin group (group 3) so like subcutaneous application of PRP, dermal application of inactive form of PRP increase flap viability by providing neovascularization from the wound bed.

Previous studies about active form of PRP and flap viability, PRP was mixed with thrombin, and calcium chloride for activation ${ }^{1,8,9}$. When PRP is activated, it become jelly form and that form isn't suitable dermal injection so in all of that studies and clinical practices, PRP applied to subcutaneous layer. In our study we didn't mix PRP with any solution for activation and we could injected that inactive form of PRP to the dermal layer because of its liquid character.

After flap elevation, plenty of ADP and AMP are formed due to ischemia ${ }^{13}$ and also plenty of noradrenalin is present due to sympathetic discharge in flap tissues. These noradrenalin and ADP cause degranulation of alfa granules and the secretion of growth factors from that granules ${ }^{14}$. Therefore, we believe that degranulation of alfa granules in inactively administered PRP was caused by noradrenalin and ADP in the dermis so flap necrosis ratio was decreased in our study.

Using autologous PRP is technically impossible for small animals as they don't have sufficient blood so we mixed blood obtained from 
different 16 rats and prepared PRP from that blood $^{15}$. During PRP separation with centrifugation as we did, leukocyte elimination from PRP content is not possible without using a cell separator so PRP contains a high concentration of leukocytes in addition to platelets ${ }^{4}$. In our study, we didn't do anything for leukocyte elimination from PRP and by using PRP obtained from different rats blood, we didn't observed any adverse effect on flap circulation and any "graft versus host" reaction. When groups with silicone placement under the flap (thereby precluding vascularization) were assessed (group 2, 4 and 6), the necrosis ratio in the flap was significantly lower in the group 6 (PRP + silicone group) than other group 2(only flap+silicone) and group 4 (saline + silicone group) and this shows that the flap viability increasing effect of PRP is caused not only via revascularization from the receiving bed. In the caudal based random pattern skin flap model ${ }^{11}$ that we used in this study, when the flap is elevated necrosis does not occur in the area close to the pedicle as there is adequate perfusion, and necrosis occurs in the ending area of the flap away from the pedicle due to ischemia. In the area called the transition zone between these areas, hyperadrenergic condition occurs due to noradrenalin released from the cut sympathetic nerves during flap elevation, and this causes an early ischemia but perfusion is restored within 12-24 hours when the hyperadrenergic condition ends ${ }^{16}$. Ischemia and the following reperfusion in this area cause ischemia-reperfusion injury $(I-R)^{3}$ and in our study we think that PRP decreases $I-R$ injury on skin flap and by that way PRP decreases necrosis ratio in flap when vascularization preclude by putting silicone layer under the flap.

\section{Conclusion}

The intradermal inactive platelet rich plasma administration increases flap viability and decreases flap necrosis.

\section{References}

1. Findikcioglu F, Findikcioglu K, Yavuzer R, Lortlar N, Atabay K. Effect of preoperative subcutaneous platelet-rich plasma and fibrin glue application on skin flap survival. Aesthetic Plast Surg. 2012 Oct;36(5):124653. doi: 10.1007/s00266-012-9954-6.

2. de Lima Silva JJ, Guimarães SB, da Silveira ER, de Vasconcelos PR, Lima GG, Torres SM, de Vasconcelos RC. Effects of Copaifera langsdorffii Desf. on ischemia-reperfusion of randomized skin flaps in rats. Aesthetic Plast Surg. 2009 Jan;33(1):104-9. doi: 10.1007/ s00266-008-9263-2.

3. van Den Heuvel MGW, Buurman WA, Bast A, van der Hulst RR. Review: ischaemiareperfusion injury in flap surgery. J Plast Reconstr Aesthet Surg. 2009 Jun;62(6):7216. doi: 10.1016/j.bjps.2009.01.060.

4. Ehrenfest DMD,Rasmusson R, Albrektsson T. Classification of platelet concentrates: from pure platelet-rich plasma (P-PRP) to leucocyte- and platelet-rich fibrin (L-PRF). Trends Biotechnol. 2009 Mar;27(3):158-67. doi: 10.1016/j.tibtech.2008.11.009.

5. Schnabel LV, Mohammed HO, Miller BJ, McDermott WG, Jacobson MS, Santangelo KS, Fortier LA. Platelet rich plasma (PRP) enhances anabolic gene expression patterns in flexor digitorum superficialis tendons. J Orthop Res. 2007 Feb;25(2):230-40. doi: 10.1002/jor.20278.

6. Marck RE, Middelkoop E, Breederveld RS. Considerations on the use of platelet-rich plasma, specifically for burn treatment. J Burn Care Res. 2014 May-Jun;35(3):219-27. doi: 10.1097/BCR.0b013e31829b334e.

7. Takikawa $M$, Nakamura $S$, Nakamura $S$, Ishirara $M$, Kishimoto $S$, Sasaki $K$, Yanagibayashi S, Azuma R, Yamamoto N, Kiyosawa T. Enhanced effect of plateletrich plasma containing a new carrier on hair growth. Dermatol Surg. 2011 Dec;37(12):1721-9. doi: 10.1111/j.15244725.2011.02123.x.

8. Li W, Enomoto $M$, Ukegawa $M$, Hirai $T$, Sotome S, Wakabayashi $Y$, Shinomiya $\mathrm{K}$, Okawa A. Subcutaneous injections 
of platelet-rich plasma into skin flaps modulate proangiogenic gene expression and improve survival rates. Plast Reconstr Surg. 2012 Apr;129(4):858-66. doi: 10.1097/ PRS.0b013e3182450ac9.

9. Wang B, Geng Q, Hu J, Shao J, Ruan J, Zheng J. Platelet-rich plasma reduces skin flap inflammatory cells infiltration and improves survival rates through induction of angiogenesis: an experiment in rabbits. J Plast Surg Hand Surg. 2016 Aug;50(4):23945. doi: 10.3109/2000656X.2016.1159216.

10.Tobita M, Uysal AC, Ogawa R, Hyakusoku H, Mizuno $\mathrm{H}$. Periodontal tissue regeneration with adipose-derived stem cells. Tissue Eng Part A. 2008 Jun;14(6):945-53. doi: 10.1089/ ten.tea.2007.0048.

11.Wang SP, Lan ZY, Xia W, Zhao X, Ma GJ, Liu $B$, Pan BH, Guo SZ. The effects of vasonatrin peptide on random pattern skin flap survival. Ann Plast Surg. 2014 Jan;72(1):94-9. doi: 10.1097/SAP.0b013e318255a3eb.

12.Kaufman T, Angel MF, Eichenlaub EH, Levin $M$, Hurwitz DJ, Futrell JW. The salutary effects of the bed on the survival of experimental flaps. Ann Plast Surg. 1985 Jan;14(1):64-73. PMID: 3883875.

13. Manson PN, Narayan KK, Im MJ, Bulkley GB, Hoopes JE. Improved survival in free skin flap transfers in rats. Surgery. 1986 Feb;99(2):211-5. PMID: 3511561.

14. Babbush CA, Kevy SV, Jacobson MS. An in vitro and in vivo evaluation of autologous platelet concentrate in oral reconstruction. Implant Dent. 2003;12(1):24-34. PMID: 12704953.

15. Conboy MJ, Conboy IM, Rando TA. Heterochronic parabiosis: historical perspective and methodological considerations for studies of aging and longevity. Aging Cell. 2013 Jun;12(3):52530. doi: 10.1111/acel.12065.

16.Cymrot $M$, Percario S, Ferreira LM. Oxidative stress and total antioxidant status in ischemic skin flaps in rats. Acta Cir Bras. 2004;19(1):18-26. doi: 10.1590/S010286502004000100003 .

\section{Correspondence:}

Erkan Orhan

Department of Plastic Surgery, Namik Kemal University

Tekirdağ Turkey

eorhan@yahoo.com

Received: Dec 07, 2016

Review: Feb 08, 2017

Accepted: March 10, 2017
Conflict of interest: none

Financial source: none 